\title{
芸在粘胶基活性炭纤维上的吸附
}

\author{
张天永 ${ }^{1,2},{ }^{*}$ 杨秋生 ${ }^{1}$ 史慧贤 $^{1}$ 韩 聪 $^{1}$ 刘 旭 ${ }^{1}$ \\ ( ${ }^{1}$ 天津大学化工学院, 天津 $300072 ; 2$ 福建省光催化重点实验室一一省部共建国家重点实验室培育基地, \\ 福州大学, 福州 350002)
}

\begin{abstract}
摘要：研究了水溶液中䓺在粘胶基活性炭纤维(VACF)吸附剂上的等温吸附. 用多种吸附方程(Langmuir、 Freundlich 、Dubinin-Radushkevitch (D-R)、Dubinin-Astalov (D-A)、Langmuir-Freundlich (L-F)、Redlich-Peterson (R-P)) 对吸附实验数据进行拟合, 考察了温度、 $\mathrm{pH}$ 和负载铜离子对荟吸附的影响. 方程拟合结果表明微孔吸附容积填 充理论对 VACF 吸附荟有较好的适用性, 优于单层吸附理论. 在 $20-40{ }^{\circ} \mathrm{C}$ 之间, 荟的吸附是自发进行的, $30{ }^{\circ} \mathrm{CW}$ 上时, 荎的吸附量显著降低. 提出了新的机理来解释 $\mathrm{pH}$ 较低时吸附量显著上升的实验现象. 负载铜离子后 VACF 对菜吸附量降低.
\end{abstract}

关键词：吸附; 活性炭纤维; 荎; 吸附方程; 铜离子 中图分类号：0647.3

\section{Adsorption of Naphthalene on a Viscose-Based Activated Carbon Fiber}

\author{
ZHANG Tian-Yong ${ }^{1,2 * *} \quad$ YANG Qiu-Sheng ${ }^{1} \quad$ SHI Hui-Xian ${ }^{1} \quad$ HAN Cong $^{1} \quad$ LIU Xu $^{1}$ \\ ( ${ }^{1}$ School of Chemical Engineering and Technology, Tianjin University, Tianjin 300072, P. R. China; \\ ${ }^{2}$ State Key Laboratory Breeding Base of Photocatalysis, Fuzhou University Fuzhou 350002, P. R. China)
}

\begin{abstract}
We studied the isothermal adsorption of naphthalene on a viscose-based activated carbon fiber (VACF) in aqueous solution. The adsorption experimental data were fitted to adsorption equations such as the Langmuir, Freundlich, Dubinin-Radushkevitch(D-R), Dubinin-Astalov(D-A), Langmuir-Freundlich (L-F), and Redlich-Peterson (R-P) equations. The effects of temperature, $\mathrm{pH}$, and pre-loaded copper ions on the VACF on the adsorption of naphthalene were investigated. The fitting results show that the micropore volume filling theory fits better than the single-layer adsorption theory for naphthalene adsorption onto VACF. The adsorption of naphthalene occurs spontaneously between 20 and $40{ }^{\circ} \mathrm{C}$. The adsorption capacity of VACF for naphthalene decreases significantly above $30{ }^{\circ} \mathrm{C}$. A new mechanism is proposed to explain why the adsorption capacity increases significantly in lower $\mathrm{pH}$ aqueous solutions. The reduction of naphthalene takes place on the VACF with pre-loaded copper ions.
\end{abstract}

Key Words: Adsorption; Activated carbon fiber; Naphthalene; Adsorption equation; Copper ion

在制焦工业、石油开采和油汽燃烧中产生了大 量的废气, 它们大都含有较多的联苯、荟、菲等多环 芳烃化合物, 最终大部分富集在水环境中. 这些多环 芳烃有机物化学性质稳定, 是人类环境致癌化学物 质中最大的一类. 目前这类物质的净化方法有生物 降解、光降解、化学絮凝、高能离子束照射、臭氧氧
化、催化分解、活性炭净化处理等. 从去除效率、成本 等方面考虑, 活性炭净化处理是较优的方法. 相比于 普通活性炭, 活性炭纤维(ACF) 具有微孔结构发达、 比表面积和吸附容量大、大量微孔开口在纤维丝表 面、孔径分布集中、对苯类化合物吸附力较强等特 点, 现已广泛用于气体分离和废水处理等方面 ${ }^{[1]}$. 由

Received: June 30, 2009; Revised: December 2, 2009; Published on Web: December 29, 2009.

*Corresponding author. Email: tyzhang@tju.edu.cn; Tel: +86-22-27406610.

The project was supported by the State Key Laboratory Breeding Base of Photocatalysis, Fuzhou University, China (K-081027).

福建省光催化重点实验室——省部共建国家重点实验室(K-081027)资助项目

C. Editorial office of Acta Physico-Chimica Sinica 
于荟具有低毒性、结构简单、在环境中易存在, 最适 合作为多芳烃的模型化合物进行研究. 同时本课题 组在研究萗一步羟基化法制备菜酚反应中, 利用 $\mathrm{ACF}$ 做为催化剂的载体, 也需要阐明荥在 ACF 上 吸附情况, 进而分析反应可能的机理 ${ }^{[2]}$. 文献对 ACF 吸附离子和强极性有机物已有报道, $\mathrm{ACF}$ 吸附离子 如 $\mathrm{CrO}_{4}^{2-} 、 \mathrm{Cu}^{2+} 、 \mathrm{Ni}^{2+} 、 \mathrm{Fe}^{2+} 、 \mathrm{Ag}^{+}$时, 溶液的 $\mathrm{pH}$ 值对吸附 量有显著影响 ${ }^{[3]}$; 吸附强极性有机物如亚甲蓝、敌草 隆、氨基三唑时, 影响因素主要有溶液 $\mathrm{pH}$ 、离子强 度、 $\mathrm{ACF}$ 的表面性质等 ${ }^{[4-5]}$. 菜作为最简单的二元芳 环的弱极性有机物, 目前鲜见其在 ACF 上液相吸附 的相关报道, 且液相吸附中, 由于存在吸附质、溶 剂、吸附剂同种分子之间及异种分子之间的竞争吸 附, 所以比气相吸附更复杂 ${ }^{[6]}$, 人们对此类吸附的机 理依然没有完全弄清楚. 本文采用粘胶基活性炭纤 维(VACF)为吸附剂, 以菜为吸附质, 研究了水溶液 中不同温度 $\mathrm{pH}$ 条件下吸附荎的等温曲线以及 $\mathrm{VACF}$ 吸附铜离子后对荎吸附的影响, 分析了影响 荟吸附的多种因素.

\section{1 实验部分}

\section{1 原料与仪器}

萗 $(\geqslant 99.5 \%)$, 江苏南通柏盛化工有限公司; $\mathrm{VACF}$, 纤维直径 15-20 $\mu \mathrm{m}$, 孔径为 1.5-2.0 nm, 总 比表面积 $1793 \mathrm{~m}^{2} \cdot \mathrm{g}^{-1}$, 其中微孔为 $1762 \mathrm{~m}^{2} \cdot \mathrm{g}^{-1}$, 鞍 山森金活性炭厂. 其它药品均为分析纯, 含量大于 99\%, 由天津市江天化工技术有限公司提供.

DF-1019 型集热式恒温磁力摚拌器(河南爱博特 科技发展有限公司); $\mathrm{He} \lambda$ ios $\gamma$ 型紫外可见分光光度 计、NICOLET380 型红外光谱仪(均为美国 Thermo); SHB-III 型循环水式真空原(郑州长城科工贸有限公 司); AR 1140 分析天平(美国 Ohaus); Autosorb-1 型全 自动比表面和孔径分布分析仪(美国 Quantachrome)

\section{$1.2 \mathrm{VACF}$ 的预处理}

将段状的 VACF 剪成 $1 \mathrm{~cm} \times 2 \mathrm{~cm}$, 加人去离子 水, 搅拌, 浸泡 $24 \mathrm{~h}$. 抽滤, 离心, 水洗 3 次. 再加人 $0.5 \mathrm{~mol} \cdot \mathrm{L}^{-1}$ 盐酸溶液, 浸泡 $24 \mathrm{~h}$. 再抽滤, 离心, 水洗 至中性, 无氯离子存在. 放人烘箱中, $120{ }^{\circ} \mathrm{C}$ 干燥 $6 \mathrm{~h}$.

\subsection{VACF 表面性质分析}

按照文献[7]方法, 采用 Boehm 滴定方法测定表 面含氧基团的数目及 PZC(等电点). 用红外光谱鉴 别 VACF 表面存在的官能团, 将干燥后的 VACF 研 为细粉末, 用 $\mathrm{KBr}$ 压片法测试红外光谱, 累加次数
为 20 次.

\section{4 吸附平衡时间测定}

称取 VACF 约 $10 \mathrm{mg}$ (精确至 $0.1 \mathrm{mg}$ ), 加人到 $250 \mathrm{~mL}$ 三角瓶中, 准确加入 $250 \mathrm{~mL}$ 的 $20.72 \mathrm{mg} \cdot \mathrm{L}^{-1}$ 菜溶液, 密封后, 在 $30{ }^{\circ} \mathrm{C}$ 恒温水浴中搅拌, 保持转速 为 $200 \mathrm{r} \cdot \mathrm{min}^{-1}$, 分别经过 $1 、 1.5 、 2 、 4 、 8 、 10 、 15 、 20$ 、 $25 、 30 \mathrm{~h}$ 后取样, 每次取样 $1 \mathrm{~mL}$, 稀释后测定其吸光 度. 并根据工作曲线方程 (以 $219.5 \mathrm{~nm}$ 作为测定波 长, 按郎伯-比尔定律得到工作曲线方程)计算其浓 度, 得到溶液浓度与时间的关系曲线.

\section{5 等温吸附曲线的测定}

\subsection{1 不同温度下的等温吸附测定}

在一系列 $250 \mathrm{~mL}$ 三角瓶中分别加人一定质量 的 VACF 和浓度为 $20.72 \mathrm{mg} \cdot \mathrm{L}^{-1}$ 的不同体积的萗 水溶液, 补加去离子水到 $250 \mathrm{~mL}$, 密封后, 在不同温 度 $\left(20 、 25 、 30 、 35 、 40{ }^{\circ} \mathrm{C}\right)$ 下于恒温水浴中搅拌 $24 \mathrm{~h}$, 保持转速为 $200 \mathrm{r} \cdot \mathrm{min}^{-1}$, 过滤, 将滤液稀释后, 测定 吸光度, 计算滤液中菜的浓度. 按下式计算吸附量: $q=V\left(C_{0}-C_{\mathrm{e}}\right) / m$, 式中, $q$ 为吸附量 $\left(\mathrm{mg} \cdot \mathrm{g}^{-1}\right), V$ 为溶液 体积 $(\mathrm{L}), C_{0}$ 和 $C_{\mathrm{e}}$ 分别为吸附前和吸附平衡时菜的 浓度 $\left(\mathrm{mg} \cdot \mathrm{L}^{-1}\right), m$ 为 VACF 的质量 $(\mathrm{g})$.

\subsection{2 不同 $\mathrm{pH}$ 的菜水溶液吸附}

在加人 VACF 前, 先用 $0.1 \mathrm{~mol} \cdot \mathrm{L}^{-1} \mathrm{NaOH}$ 溶液 或盐酸溶液调节荥溶液的 $\mathrm{pH}$ 为碱性或酸性, 吸附 平衡后, 再测定溶液的 $\mathrm{pH}$. 其余同 1.5.1 节.

\subsubsection{VACF-CU 对菜的吸附}

将 VACF 换成 VACF-Cu, 在 $30{ }^{\circ} \mathrm{C}$ 下按 1.5.1 节 的方法吸附. VACF-Cu 是将预处理后的 $6 \mathrm{~g} \mathrm{VACF}$ 浸泡在 $90 \mathrm{~mL}$ 的 $0.40 \mathrm{~mol} \cdot \mathrm{L}^{-1}$ 的硝酸铜溶液中, 室 温下保持 $24 \mathrm{~h}$, 过滤后, 洗至滤液中无铜离子为止. $120{ }^{\circ} \mathrm{C}$ 干燥 $6 \mathrm{~h}$, 用电感耦合等离子体原子发射光 谱仪(ICP)测定铜离子负载量为 $0.62 \mathrm{mmol} \cdot \mathrm{g}^{-1}$, 记为 $\mathrm{VACF}-\mathrm{Cu}$.

\section{2 结果与讨论}

\subsection{VACF 的表征}

活性炭纤维主要有粘胶基、聚丙烯腈基、酚醛 基、沥青基四类. VACF 主要由 $\mathrm{C} 、 \mathrm{H} 、 \mathrm{O}$ 三种元素组 成, 含有许多不同含氧的官能团结构, 如羧基、羰基、 内酯基、酚羟基、醌基等. 不同强度的碱可以与相应 强度的表面酸性基团反应, 经 Boehm 滴定 ${ }^{[7]}$ 测得总 酸为 $1.26 \mathrm{mmol} \cdot \mathrm{g}^{-1}$, 羧基为 $0.44 \mathrm{mmol} \cdot \mathrm{g}^{-1}$, 内酯酸 为 $0.11 \mathrm{mmol} \cdot \mathrm{g}^{-1}$, 酚差基为 $0.71 \mathrm{mmol} \cdot \mathrm{g}^{-1}, \mathrm{PZC}$ 为 


\subsection{6 .}

VACF 的表面含氧官能团较多, 红外光谱中峰 形叠加严重. 图 1 中, $3440 \mathrm{~cm}^{-1}$ 为 $\mathrm{VACF}$ 最强吸收 峰, 表示存在大量不同聚合程度的羟基, 这些物质可 能是少量的吸附水分和醇酚的多聚物. $1645 \mathrm{~cm}^{-1}$ 为 乱层石墨的类芳环结构峰和羧基的反对称伸缩振动 峰. $1100 \mathrm{~cm}^{-1}$ 处为酚羟基的变角振动吸收峰和醚键 的反对称吸收峰. $1150 \mathrm{~cm}^{-1}$ 为内酯基的反对称伸缩 峰 ${ }^{[8-10]}$. 通过以上两种表征方法的相互补充及印证, 较清楚地了解 VACF 的表面官能团情况.

\section{2 吸附平衡时间}

以 $219.5 \mathrm{~nm}$ 为检测波长, 测得不同浓度下荎 的吸光度数据, 经过 Origin7.5 处理, 得到线性方程 为 $C=2.2611 A+0.1333$, 式中 $A$ 为测得的䒬溶液的 吸光度数值, $C$ 为萘溶液的浓度 $\left(\mathrm{mg} \cdot \mathrm{L}^{-1}\right)$, 相关系数 $r=0.999$.

水溶液中有机物在 VACF 上吸附达到平衡的 时间, 主要由浓度、温度、摚拌情况、溶解度、吸附剂 性质等多种因素决定. 苯类物质的吸附动力学模型 多为类二级 ${ }^{[11]}$, 吸附质浓度越高, 吸附速度越快, 吸 附质浓度随时间呈非线性变化, 但是初始吸附时呈 现一定的线性关系(见图 2), 可能是吸附质浓度远远 大于空白吸附位的原因. 从图 2 可知, 在 $30{ }^{\circ} \mathrm{C}, 200$ $\mathrm{r} \cdot \mathrm{min}^{-1}$ 情况下, 达到吸附平衡的时间为 $15 \mathrm{~h}$. 因此 设定了 $24 \mathrm{~h}$ 的足够时间来保证达到吸附平衡.

\section{3 等温吸附方程}

\subsection{1 不同的拟合方程}

等温吸附曲线常用来表征不同浓度下吸附的平 衡状态, 同时也可以得到吸附剂孔结构, 吸附热效应 等信息. 图 3 为 $30{ }^{\circ} \mathrm{C}$ 时, 不同浓度的荎溶液吸附 24 $h$ 时, 所得到的荎的吸附等温线.

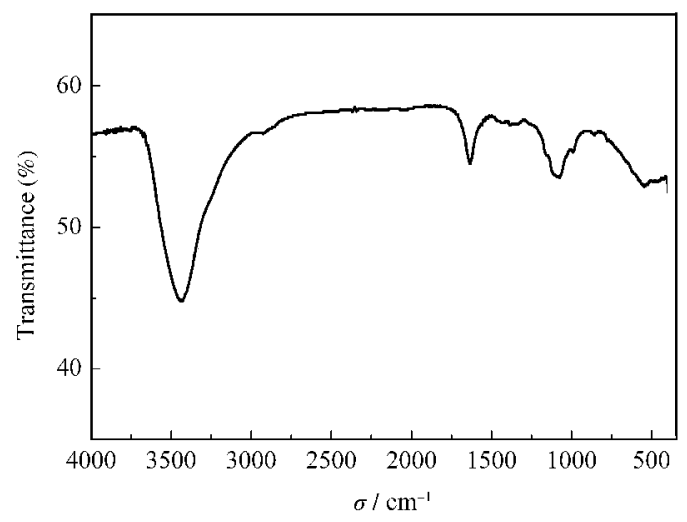

图 $1 \mathrm{VACF}$ 的红外光谱

Fig.1 Infrared spectrum of VACF

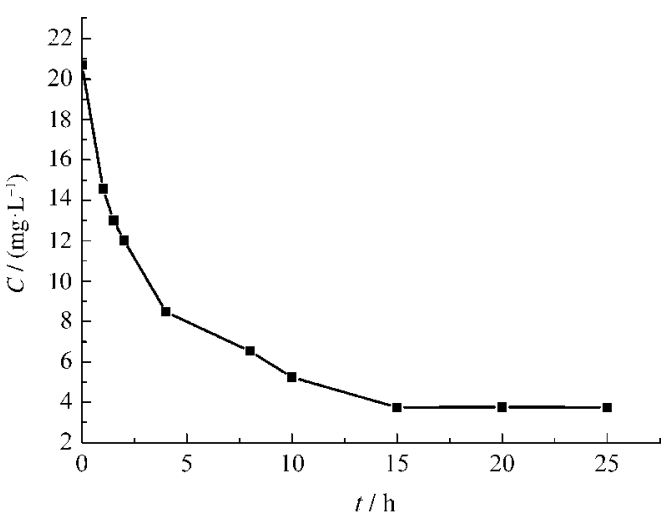

图 2 VACF 对萗吸附的浓度-时间关系

Fig.2 Concentration-time $(C-t)$ in adsorption of naphthalene on VACF

参考了许多等温吸附方程的报道 ${ }^{[12]}$, 采用 Origin 7.5 对实验数据(见图 3)按表 1 中的方程进行了拟 合. 结果表明, 后三个方程(D-A、L-F、R-P)要优于 前三个方程(Langmuir、Freundlich、D-R). Freundlich 方程是经验式, 主要用来拟合异质表面. 拟合结果较 差,也表明 VACF 表面的均质性相对较好, $n$ 值较大 也表示芸与 VACF 之间的吸附力较强. Langmuir 方 程是基于单分子层吸附, 吸附质之间无作用力, 吸附 剂表面均一的基础而建立的. 菜分子的大小为 0.7 $\mathrm{nm}^{[13]}$, 面积约为 $0.45 \mathrm{~nm}^{2}$, 按菜饱和吸附量 $763.8 \mathrm{mg}$. $\mathrm{g}^{-1}$ 计算, 达到吸附平衡时荎约占 VACF 总比表面积 的 $90 \%$ 左右, 说明菜在 VACF 上吸附效果较佳. 这 与 VACF 是非极性表面, 较小的孔径结构特点密切 相关. 荎与 VACF 表面之间的色散力起主要作用 ${ }^{[14-15]}$. Langmuir 方程拟合结果表明荟在 VACF 的吸附接

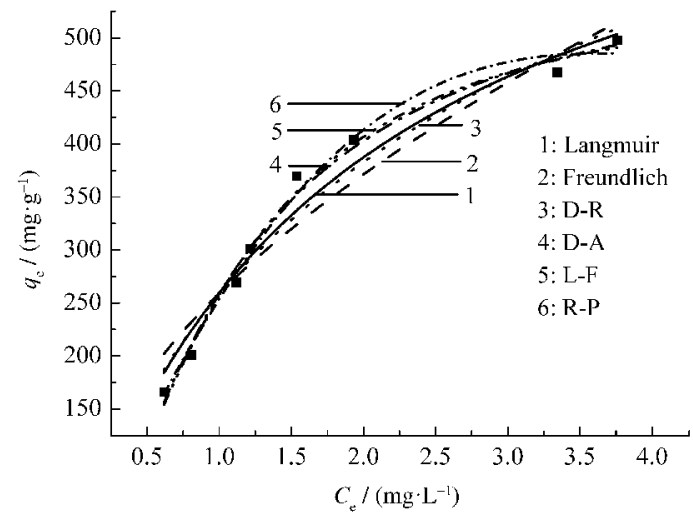

图 $330{ }^{\circ} \mathrm{C}$ 时菜在 $\mathrm{VACF}$ 上的等温吸附及其拟合曲线 Fig.3 Isotherm and fitting-curve in adsorption of naphthalene on VACF at $30{ }^{\circ} \mathrm{C}$

D-R: Dubinin-Radushkevitch, D-A: Dubinin-Astalov, L-F: Langmuir-Freundlich, R-P: Redlich-Peterson 
表 $130{ }^{\circ} \mathrm{C}$ 时不同等温吸附方程的拟合结果

Table 1 Fitting results of different isotherm equations at $30{ }^{\circ} \mathrm{C}$

\begin{tabular}{llll}
\hline & \multicolumn{1}{c}{ Isotherm equation } & \multicolumn{1}{c}{ Parameters } & $R^{2}$ \\
\hline Freundlich & $q_{\mathrm{e}}=K_{\mathrm{F}} C_{\mathrm{e}}^{1 / n}$ & $K_{\mathrm{F}}=259.4\left(\mathrm{mg} \cdot \mathrm{g}^{-1}\right) /\left(\mathrm{mg} \cdot \mathrm{L}^{-1}\right)^{0.52}, 1 / n=0.52$ & 0.934 \\
Langmuir & $q_{\mathrm{e}}=K_{\mathrm{L}} q_{\mathrm{m}} C_{\mathrm{e}} /\left(1+K_{\mathrm{L}} C_{\mathrm{e}}\right)$ & $q_{\mathrm{m}}=763.8 \mathrm{mg} \cdot \mathrm{g}^{-1}, K_{\mathrm{L}}=0.51 \mathrm{~L} \cdot \mathrm{mg}^{-1}$ & 0.972 \\
D-R & $q_{\mathrm{e}}=q_{\mathrm{m}} \exp \left\{-\left[(R T / E) \ln \left(C_{\mathrm{s}} C_{\mathrm{e}}\right)\right]^{2}\right\}$ & $q_{\mathrm{m}}=825.3 \mathrm{mg} \cdot \mathrm{g}^{-1}, E=8.71 \mathrm{~kJ} \cdot \mathrm{mol}^{-1}$ & 0.962 \\
D-A & $q_{\mathrm{e}}=q_{\mathrm{m}} \exp \left\{-\left[(R T / E) \ln \left(C_{\mathrm{s}} C_{\mathrm{e}}\right)\right]^{4}\right\}$ & $q_{\mathrm{m}}=566.6 \mathrm{mg} \cdot \mathrm{g}^{-1}, E=9.98 \mathrm{~kJ} \cdot \mathrm{mol}^{-1}$ & 0.990 \\
L-F & $q_{\mathrm{e}}=q_{\mathrm{m}}\left(K_{\mathrm{LF}} C_{\mathrm{e}}\right)^{m} /\left[1+\left(K_{\mathrm{LF}} C_{\mathrm{e}}\right)^{m}\right]$ & $q_{\mathrm{m}}=551.4 \mathrm{mg} \cdot \mathrm{g}^{-1}, K_{\mathrm{LF}}=0.92 \mathrm{~L} \cdot \mathrm{mg}^{-1}, m=1.68$ & 0.992 \\
R-P & $q_{\mathrm{e}}=K_{\mathrm{R}} C_{\mathrm{e}} /\left(1+a C_{\mathrm{e}}^{\beta}\right)$ & $K_{\mathrm{R}}=275.4 \mathrm{~L} \cdot \mathrm{g}^{-1}, a=0.086 \mathrm{~L} \cdot \mathrm{mg}^{-1}, \beta=1.943$ & 0.991 \\
\hline
\end{tabular}

近单层吸附, 但是由于表面存在含氧官能团和荂溶 液溶度较高, 所以偏离理想状态略显偏大, 导致拟合 结果出现偏差. 进一步研究发现 Langmuir 方程在结 合了 Freundlich 方程特点后形成的 L-F 和 R-P 三参 数方程, 拟合结果均优良.

D-A 和 D-R 方程的理论基础是微孔吸附容积 充填理论 ${ }^{[16}$. 活性炭纤维是乱层石墨结构, 孔径多在 $2 \mathrm{~nm}$ 以下, 具有极大的表面能, 微孔与孔壁共同作 用形成强大的分子场, 提供了一个吸附态分子物理 和化学变化的准高压体系, 被吸附分子按吸附势大 小逐步充填. 荎的吸附主要在 $2 \mathrm{~nm}$ 以下的孔中进 行 ${ }^{[14,17]}$, 此种理论与 VACF 的结构特点较符合, 拟合 结果也较好. 当用 Weibull 分布(D-A)取代 Gaussian 分布(D-R)来描述孔的分布特点, 方程指数由 2 变为 4 时, D-A 方程对菜在 VACF 上的等温吸附曲线拟 合效果更佳.

\subsection{2 温度对菜吸附的影响}

萗为芳烃类物质, 性质稳定, 环上没有被取代 的极性基团, 在非极性面上主要是物理吸附 ${ }^{[19]}$. 参 照文献[20]的方法, 得到活性炭吸附菜的标准吸附 自由能 $\Delta G$ 的公式为 $\Delta G=-R T \ln \left(q_{\mathrm{m}} K_{\mathrm{L}} / S d\right)$, 其中 $S$ 为 $\mathrm{VACF}$ 的比表面积, $d$ 为吸附层厚度, 取值为 $0.6 \mathrm{~nm}$. 经计算, $20-40{ }^{\circ} \mathrm{C}$ 之间菜的吸附自由能 $\Delta G$ 约为 -32 $\mathrm{kJ} \cdot \mathrm{mol}^{-1}$, 小于 0 , 说明吸附为自发进行.

表 2 列出了不同温度下菜在 VACF 上吸附拟 合 Langmuir 方程. 表 2 中的数据表明, $20-30{ }^{\circ} \mathrm{C}$ 之 间 VACF 的饱和吸附量变化不大, 说明微孔吸附接
近饱和后, 很难进行再吸附. 色散力与温度变化无 相关性, 所以进一步证明了色散力为主要的吸附作 用力 ${ }^{[21]}$. 但是随着温度的上升, 菜在水中的溶解度 增大, 吸附的分子动能增加, 同时官能团之间的静电 作用也下降, 水簇被破坏, 水分子可以自由进入微孔 中, 导致平衡吸附量下降. 温度 $30 、 35 、 40{ }^{\circ} \mathrm{C}$ 时, 平衡 吸附量分别为 $763.8 、 676.8 、 404.2 \mathrm{mg} \cdot \mathrm{g}^{-1}$, 变化显著, 可能是 VACF 某类官能团集体作用的结果. 这一点 有待于进一步的研究分析.

\subsection{3 pH 对菜吸附的影响}

在水溶液中的吸附, 由于存在大量的水分子, 它 与VACF、吸附质之间存在不同的作用力, 所以比氮 气、烷烃之类的气体吸附情况更复杂. 大量的实验和 分子模拟研究表明: 水分子在非极性孔中的存在方 式, 主要与温度、压力、吸附剂的表面官能团、 $\mathrm{pH}$ 等 有关 ${ }^{[22]}$. 单组份吸附质水在 VACF 上吸附时, 水分子 主要以水簇方式存在. 水分子首先吸附在吸附剂的 表面官能团上, 然后以吸附的水分子为第二吸附位, 再通过氢键方式吸附水分子, 最终形成 5-10 个分 子的水簇. 另一方面, 水分子形成水簇后, 偶极矩降 低, 也有利于直接与非极性面接触 ${ }^{[23-25]}$. 吸附质同时 存在水与萮时, 菜与 VACF 非极性表面的作用力较 强, 替代了部分吸附的水分子, 使孔径变窄, 且水分 子与吸附的芸分子之间的距离较水分子之间的大, 二者都不利于水分子在孔中形成稳定三维结构的 氢键和降低偶极矩, 最终导致水分子在微孔内的吸 附量减少 ${ }^{[26]}$; 在孔口附近的含氧官能团, 主要以羧基

表 2 不同温度下菜在 VACF 上吸附拟合 Langmuir 方程

Table 2 Fitting-equation in adsorption of naphthalene on VACF at different temperatures

\begin{tabular}{ccccc}
\hline$T /{ }^{\circ} \mathrm{C}$ & Fitting-eqaution & $R^{2}$ & Naphthalene solubility in $1000 \mathrm{~g}$ water $(\mathrm{mg})^{*}$ & $\Delta G /\left(\mathrm{kJ} \cdot \mathrm{mol}^{-1}\right)$ \\
\hline 20 & $q_{\mathrm{e}}=796.3 \times 0.93 C_{\mathrm{e}}\left(1+0.93 C_{\mathrm{e}}\right)$ & 0.982 & 28.7 & -32.7 \\
25 & $q_{\mathrm{e}}=775.5 \times 0.88 C_{\mathrm{e}}\left(1+0.88 C_{\mathrm{e}}\right)$ & 0.974 & 33.7 & -33.1 \\
30 & $q_{\mathrm{e}}=763.8 \times 0.51 C_{d}\left(1+0.51 C_{\mathrm{e}}\right)$ & 0.972 & 41.5 & -32.2 \\
35 & $q_{\mathrm{e}}=676.8 \times 0.26 C_{d}\left(1+0.26 C_{\mathrm{e}}\right)$ & 0.967 & 47.5 & -30.7 \\
40 & $q_{\mathrm{e}}=404.2 \times 1.31 C_{d}\left(1+1.31 C_{\mathrm{e}}\right)$ & 0.975 & 55.8 & -34.1 \\
\hline \multicolumn{5}{c}{}
\end{tabular}


为初始吸附位点, 形成水簇大分子, 阻挡萗的吸附 ${ }^{[27]}$.

$\mathrm{pH}$ 影响吸附质的溶解度、吸附质与吸附剂之间 色散力和静电力 ${ }^{[28]}$. 静电力主要产生在吸附质和吸 附剂发生解离的情况下. 色散力的机理主要有 $\pi-\pi$ 色散机理、氢键机理、电子供受复合物机理 ${ }^{[29]}$. 不同 性质的活性炭吸附苯或荎受 $\mathrm{pH}$ 影响不同, 有的随 $\mathrm{pH}$ 升高而降低 ${ }^{[30]}$, 相反的情况也会存在 ${ }^{[31]}$, 而且有 的吸附量受 $\mathrm{pH}$ 影响不大 ${ }^{[32]}$. 在本实验条件下, 当 $\mathrm{pH}$ 为 12.67 和 5.61 时, 荎的吸附量差别不大, 而 $\mathrm{pH}$ 为 2.52 时吸附量显著上升(图 4).

$\mathrm{pH}$ 为 5.61 时, 菜的吸附面积占总 VACF 表面 积的 $90 \%$, 荎的竞争吸附能力要远大于水分子. 但 是在 $\mathrm{pH}$ 偏离 $\mathrm{PZC}$ 较大时, 表面含氧基团形成的氢 键受静电力作用影响较大 ${ }^{[33]}, \mathrm{pH}$ 降低时, 一种作用 是表面含氧基团结合的氢离子增多导致碳表面的电 子密度下降, 萗与炭表面的色散力减弱, 导致吸附作 用减弱, 另一种作用是氢离子具有强酸性, 吸附在炭 表面上相关碱性基团处, 菜分子也具有一定的碱 性 ${ }^{[30]}$, 吸附的氢离子通过与荎环上的 $\pi$ 键发生非典 型的氢键作用, 在炭表面发生荟溶液的浓集作用 ${ }^{[34]}$, 使菜分子易进人到微孔中进行填充, 在适当大小的 微孔中通过萗环之间的 $\pi-\pi$ 色散作用形成菜的二 聚物或三聚物 ${ }^{[35]}$, 使吸附量上升. 炭表面的含氧基团 的种类、数量和位置决定了第一种作用的大小, 氢离 子浓度与第二种作用密切相关, 吸附量的增加或减 少取决定于这两种作用的对比.

$\mathrm{pH}$ 为 12.67 时, 过量的氢氧根作用下, $\mathrm{VACF}$ 表面具有少量的负电荷, 水分子可能从二聚物形成 五聚物 ${ }^{[36]}$, 阻挡萗分子进入微孔中, 但作用很弱. 荎 仍保持分子状态, 未发生解离, 静电斥力也较弱, 此

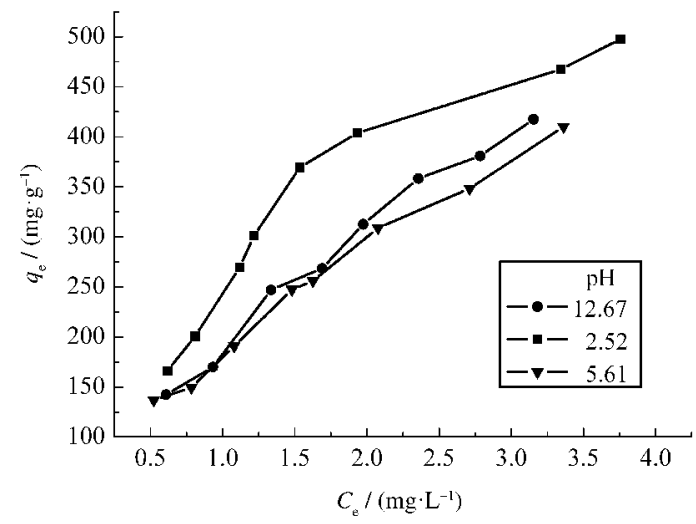

图 4 不同 $\mathrm{pH}$ 下萗的等温吸附

Fig.4 Isotherm adsorption of naphthalene on VACF at different $\mathbf{p H}$ values

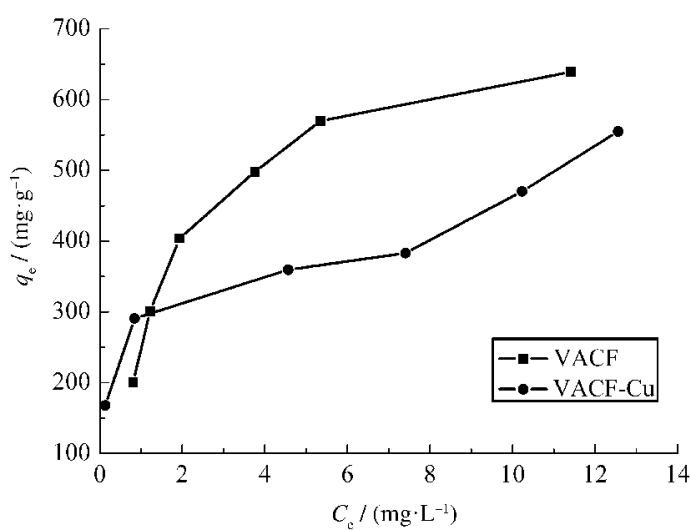

图 5 萗在 VACF 和 VACF-Cu 上的等温吸附

Fig.5 Isotherm adsorption of naphthalene on VACF and VACF-Cu

时 $\pi-\pi$ 色散力仍是吸附的主导因素. 菜在 VACF 上的吸附量变化不大.

\subsection{4 负载铜离子对吸附的影响}

铜离子具有一定的氧化性, 在催化氧化反应中 应用广泛 ${ }^{[37]}$. 铜离子能够发生类 Fenton 反应, 对荎 环进行催化差基化反应. 在进行反应时, 先将金属离 子吸附到活性炭上, 再对相关物质进行吸附, 最后再 进行表面的催化反应, 所以吸附作用对催化有重要 影响.

吸附金属离子后的活性炭与原炭对比, 常出现 被吸附物的吸附量的增大或减少 ${ }^{[38]}$. 吸附铜离子后 的 VACF-Cu, 与原 VACF 相比, 䓫的吸附量降低(图 5). 这是由于吸附在 VACF 表面的铜离子, 在局部表 面氧不能完全满足全部的配位数时, 会同时吸附水 分子, 形成较大的水合离子簇, 一方面使 VACF 表 面极性增加, 不利于非极性的荎吸附, 同时阻挡荟分 子进人微孔. 这与在非极性溶剂中 $\mathrm{Y}$ 分子篮通过裸 露的阳离子与荟环上 $\pi$ 键作用形成稳定的二聚物 是完全不同的 ${ }^{[3]}$. 从吸附曲线上看, $\mathrm{VACF}-\mathrm{Cu}$ 的吸 附曲线由原来的 VACF 的 I 型向 II 型转变, 也说明 部分微孔被阻塞, 大孔百分比数量增加.

\section{3 结 论}

VACF 具有类分子笁样的微孔结构, 本文研究 了其在水溶液中对荎的等温吸附. 通过对荎吸附曲 线的拟合, 说明微孔吸附容积充填理论对 VACF 的 适用性; 结果也说明 VACF 吸附水溶液中荎是自发 进行的, 温度对吸附量的影响不具有线性关系, 溶液 温度 $30{ }^{\circ} \mathrm{C}$ 以上时, 吸附量显著降低. 重点分析了水 对漛吸附的竞争影响, 同中性条件下荎的吸附相比, 
酸性与碱性表现出明显不同的吸附量变化, 对酸性 条件下吸附量显著增长提出了新的机理. 作为催化 剂载体的 VACF, 预吸附铜离子后, 会导致荎的吸附 量降低, VACF-Cu 的吸附曲线, 由原来的 VACF 的 I 型向 II 型转变. 通过对荎液相吸附的研究, 可以促 进 ACF 在多环芳烃净化处理方面的应用, 同时对萘 催化差基化制备荎酚的机理研究方面有所贡献.

\section{References}

1 Pan, H. Y.; Li, Z.; Xia, Q. B.; Xi, H. X; Li, J. Journal of Functional Materials, 2008, 39: 324 [潘红艳, 李 忠, 夏启斌, 奚红霞, 李 晶. 功能材料, 2008, 39: 324]

2 Zhang, T. Y.; Yang, Q. S.; Lu, Z.; Liu, X.; Wang, Z.; Li, X. X. Chemical Industry and Engineering Progress, 2009, 28: 55 [张天永, 杨秋生, 卢 洲, 刘 旭, 王 昭, 李晓旭. 化工进展, 2009, 28: 55]

3 Ko, Y. G.; Choi, U. S. Separation and Purification Technology, 2007, 57: 338

4 Fontecha-Cáamara, M. A.; López-Ramón, M. V.; Álvarez-Merino, M. A.; Moreno-Castilla, C. Langmuir, 2007, 23: 1242

5 Pelekani, C.; Snoeyink, V. L. Carbon, 2000, 38: 1423

6 Qi, S. Y.; Hay, K. J.; Cal, M. P. Advances in Environmental Research, 2000, 4: 357

7 Boehm, H. P. Carbon, 1994, 32: 759

8 Salame, I. I.; Bandosz, T. J. J. Colloid Interf. Sci., 2001, 240: 252

9 Huang, Q.; Huang, Y. Q.; Pan, D. Journal of Donghua University, 2004, 21: 57

10 Ning, Y. C. Structural identification of organic compounds and organic spectroscopy. 2nd ed. Beijing: Science Press, 2000: 322370 [宁永成. 有机化合物结构鉴定与有机波谱学(第二版). 北 京: 科学出版社, 2000: 322-370]

11 Srivastava, V. C.; Swamy, M. M.; Mall, I. D.; Prasad, B.; Mishra, I. M. Colloids Surf. A, 2006, 272: 89

12 Li, K.; Zheng, Z.; Huang, X.; Zhao, G.; Feng, J.; Zhang, J. J. Hazard. Mater., 2009, 166: 213

13 Kao, J.; Allinger, N. L. J. Am. Chem. Soc., 1977, 99: 975

14 Ahn, C. K.; Kim, Y. M.; Woo, S. H.; Park, J. M. Chemosphere, 2007, 69: 1681

15 Ania, C. O.; Cabal, B.; Pevida, C.; Arenillas, A.; Parra, J. B.; Rubiera, F.; Pis, J. J. Water Res., 2007, 41: 333

16 Wu, J. F.; Strömqvist, M. E.; Claesson, O.; Fängmark, I. E.;
Hammarström, L. G. Carbon, 2002, 40: 2587

17 Ania, C. O.; Cabal, B.; Pevida, C.; Arenillas, A.; Parra, J. B.; Rubiera, F.; Pis, J. J. Appl. Surf. Sci., 2007, 253: 5741

18 Miller, D. J.; Hawthorne, S. B. Anal. Chem., 1998, 70: 1618

19 Seredych, M. M.; Guńko, V. M.; Gierak, A. Appl. Surf. Sci., 2005, 242: 154

20 Sun, M. L.; Cheng, R. M.; Xu, X. C.; Chen, Y. W.; Li, M. G. Chemical Research and Application, 2006, 18: 13 [孙明礼, 成荣 明, 徐学诚, 陈奕卫, 李茂刚. 化学研究与应用, 2006, 18: 13]

21 García-Araya, J. F.; Beltrán, F. J.; Álvarez, P.; Masa, F. J. Adsorption, 2003, 9: 107

22 Qi, N.; LeVan, M. D. Ind. Eng. Chem. Res., 2005, 44: 3733

23 Ohba, T.; Kanoh, H.; Kaneko, K. J. Phys. Chem. B, 2004, 108 14964

24 Striolo, A.; Gubbins, K. E.; Chialvo, A. A.; Cummings, P. T. Mol. Phys., 2004, 102: 243

25 Fletcher, A. J.; Uygur, Y.; Thomas, K. M. J. Phys. Chem. C, 2007, 111: 8349

26 Easton, E. B.; Machin, W. D. J. Colloid Interf. Sci., 2000, 231: 204

27 Rablen, P. R.; Lockman, J. W.; Jorgensen, W. L. J. Phys. Chem. A, 1998, 102: 3782

28 Pastrana-Martínez, L. M.; López-Ramón, M. V.; Moreno-Castilla, C. J. Colloid Interf. Sci., 2009, 331: 2

29 Villacañas, F.; Pereira, M. F. R.; Órfão, J. J. M.; Figueiredo, J. L. J. Colloid Interf. Sci., 2006, 293: 128

30 Terzyk, A. P.; Cwiertnia, M. S.; Wisniewski, M.; Gauden, P. A.; Rychlicki, G.; Szymanski, G. S. Appl. Surf. Sci., 2007, 253: 4006

31 Wibowo, N.; Setyadhi, L.; Wibowo, D.; Setiawan, J.; Ismadji, S. J. Hazard. Mater., 2007, 146: 237

32 Zhu, X. D.; Liang, H. D. Science Technology and Engineering, 2007, 7: 2205 [朱仙弟, 梁华定. 科学技术与工程, 2007, 7: 2205]

33 Mignon, P.; Loverix, S.; Geerlings, P. Chem. Phys. Lett., 2005, 401: 40

34 Thomas, K. J.; Sunoj, R. B.; Chandrasekhar, J.; Ramamurthy, V. Langmuir, 2000, 16: 4912

35 Tsuzuki, S.; Honda, K.; Uchimaru, T.; Mikami, M. J. Chem. Phys., 2004, 120: 647

36 Kim, J.; Suh, S. B.; Kim, K. S. J. Chem. Phys., 1999, 111: 10077

37 Lu, C. Y.; Wey, M. Y.; Chen, L. I. Appl. Catal. A, 2007, 325: 163

38 Somy, A.; Mehrnia, M. R.; Amrei, H. D.; Ghanizadeh, A.; Safari, M. Int. J. Greenhouse Gas Control, 2009, 3: 249 